\title{
Abelian integrals in holomorphic foliations
}

Hossein Movasati

\begin{abstract}
The aim of this paper is to introduce the theory of Abelian integrals for holomorphic foliations in a complex manifold of dimension two. We will show the importance of Picard-Lefschetz theory and the classification of relatively exact 1-forms in this theory. As an application we identify some irreducible components of the space of holomorphic foliations of a fixed degree and with a center singularity in the projective space of dimension two. Also we calculate higher Melnikov functions under some generic conditions.
\end{abstract}

\section{Introduction}

Let us be given a differential equation

$$
\frac{d y}{d x}=\frac{P(x, y)}{Q(x, y)}
$$

in the real plane $\mathbb{R}^{2}$, where $P$ and $Q$ are two polynomials in $x$ and $y$. Let $H(P, Q)$ denote the number of limit cycles of the above differential equation and

$$
H_{n}=\max \{H(P, Q) \mid \operatorname{deg}(P), \operatorname{deg}(Q) \leq n\}
$$

For many years it was believed that the finiteness of $H(P, Q)$ have been proved by Dulac in $[\mathrm{Du}]$. But it was found that $[\mathrm{Du}]$ contains a gap and then the complete proof was given in [Il1] and [Ec] independently. Nowadays the question of whether $H_{n}$ is finite or not is considered as the Hilbert sixteen problem in differential equations. An equation (1.1) has a first integral or is called integrable if there are two polynomials $F$ and $G$ in $\mathbb{R}^{2}$ such that $\frac{F}{G}$ is

2000 Mathematics Subject Classification: 57R30, 14D99, 32G34.

Keywords: Holomorphic foliations, holonomy, Picard-Lefschetz theory. 
constant on its solutions. In this case the equation (after reducing) has the form

$$
\frac{d y}{d x}=-\frac{G F_{x}-F G_{x}}{G F_{y}-F G_{y}}
$$

When $G \equiv 1$ then the equation is called Hamiltonian. Let $\mathcal{S}_{n}^{\prime}\left(\operatorname{resp} . \mathcal{S}_{n}\right)$ denote the space of equations (1.1) defined by real (resp. complex) polynomials $P$ and $Q \operatorname{deg}(P), \operatorname{deg}(Q) \leq n$. The space $\mathcal{S}_{n}^{\prime}$ is parameterized by the coefficients of $P$ and $Q$.

One of the first attempts to solve the mentioned problem was made by two Russian mathematicians I. G. Petrovskiü and E. M. Landis ([LP1],[LP2]). They complexified the equation (1.1) and considered the equation in $\mathbb{C}^{2}$ and tried to find another set of cycles $\mathcal{C}(P, Q)$ in the solutions of (1.1) whose cardinality is not less than the number of limit cycles in the related real equation. Then they tried to calculate a uniform upper bound for the cardinality of $\mathcal{C}(P, Q)$ (and hence $H_{n}$ ) by perturbation of integrable equations. Let $\left\{\delta_{t}\right\}_{t \in(\mathbb{R}, 0)}$ be a continuous family of closed solutions of an integrable equation. They observed that the bifurcation of limit cycles from this family of closed cycles is related with the zeros of a certain Abelian integrals.

Although their work had errors, the idea of using Abelian integrals to count the number of limit cycles of a perturbed Hamiltonian equation has been one of the useful tools in getting approach to the Hilbert Sixteen Problem. For this see the book [Ro] and its references. The aim of this text is to introduce an algebro-geometric approach to these Abelian integrals. Instead of $\mathbb{C}^{2}$ we consider an arbitrary two dimensional compact complex manifolds and instead of Hamiltonian fibrations we consider the fibrations of meromorphic functions on $M$. In section 2 and 3 holomorphic foliations and the Picard-Lefschetz theory of meromorphic functions on $M$ are introduced. In section 4 we see how Abelian integrals appear in the formula of the first Melnikov function. In section 5 we will encounter with relatively exact 1-forms. In section 6 we will classify some irreducible components of holomorphic foliations in $\mathbb{P}^{2}$ with a center singularity and finally in section 7 we will calculate higher order Melnikov functions under some generic conditions.

This work started from my doctorate thesis. I want to use this opportunity to express my thanks to my advisor Alcides Lins Neto, who introduced me with the article [Il]. Also, thanks go to Cesar Camacho and Paulo Sad my teachers in IMPA-Brazil. I thank also S. Shahshahani who made my visiting from IPM-Iran possible when I was working on some parts of this work. I thank also MPIM-Germany which the final version of this paper was obtained there. 


\section{Holomorphic Foliations}

Let $M$ be a compact complex manifold of dimension two. A holomorphic foliation in $M$ with isolated singularities is given by a collection of holomorphic 1-forms $\omega_{\alpha}$ defined on $U_{\alpha}, \alpha \in I$, where $\left\{U_{\alpha}\right\}_{\alpha \in I}$ is an open covering of $M$, and such that

$$
\omega_{\alpha}=g_{\alpha \beta} \omega_{\beta}, \alpha, \beta \in I
$$

where $g_{\alpha \beta}$ is a holomorphic without zero function in $U_{\alpha} \cap U_{\beta}$. Furthermore we assume that the set of points in which $\omega_{\alpha}$ is zero has codimension greater than one (discrete set). In other words $\omega_{\alpha}$ has not zero divisor.

Therefore for any foliation $\mathcal{F}$ there is associated a line bundle $L$ given by the transition functions $\left\{g_{\alpha \beta}\right\}_{\alpha, \beta \in I} \in H^{1}\left(M, \mathcal{O}^{*}\right)=\operatorname{Pic}(M)$. We also say that the foliation $\mathcal{F}$ is of degree $L$. The data (2.1) can be considered as a holomorphic section $\omega \in H^{0}\left(M, \Omega^{1} \otimes L\right)$ without zero divisor, where $\Omega^{1}$ is the cotangent bundle of $M$. Since $M$ is compact, $H^{0}\left(M, \Omega^{1} \otimes L\right)$ is a finite dimensional space (this is a simple corollary of Grauert direct image theorem). By $\mathcal{F}(\omega)$ we mean that the foliation $\mathcal{F}$ is given by the 1 -form $\omega \in H^{0}\left(M, \Omega^{1} \otimes L\right)$. It is easy to check that for any foliation $\mathcal{F}$ there exists a unique line bundle $L$ in $M$ such that $\mathcal{F}$ is given by a holomorphic without zero divisor element of $H^{0}\left(M, \Omega^{1} \otimes L\right)$. From now on we fix a line bundle $L$ and assume that $H^{0}\left(M, \Omega^{1} \otimes L\right)$ has a holomorphic without zero divisor section. In this case the set of 1 -forms $\omega \in H^{0}\left(M, \Omega^{1} \otimes L\right)$ without zero divisor is an open subset of $H^{0}\left(M, \Omega^{1} \otimes L\right)$.

If $\omega \in H^{0}\left(M, \Omega^{1} \otimes L\right)$ has a zero divisor we use the following trick: Let $\omega \in H^{0}\left(M, \Omega^{1} \otimes L\right)$ be a holomorphic section with the zero divisor $Z$. Let $L_{Z}$ be the line bundle associated to $Z$ and $s \in H^{0}\left(M, L_{Z}\right)$ be the holomorphic section with the zero divisor $Z$. Now $\frac{\omega}{s}$ is a holomorphic without zero divisor section of $H^{0}\left(M, \Omega \wedge L \wedge L_{Z}^{-1}\right)$, and so, we can substitute $L$ for $L \wedge L_{Z}^{-1}$.

Two holomorphic without zero divisor sections $\omega, \omega^{\prime} \in H^{0}\left(M, \Omega^{1} \otimes L\right)$ induce the same foliation if and only if $\omega=c . \omega^{\prime}$, where $c$ is a constant. Therefore the space of foliations of degree $L$, namely $\mathcal{F}(M, L)$, form an open subset of the projective space

$$
\operatorname{Proj}\left(H^{0}\left(M, \Omega^{1} \otimes L\right)\right)
$$

Many times we need the foliation $\mathcal{F}$ to be given by a meromorphic 1-form in $M$. In these cases we assume that $H^{0}(M, L) \neq 0$ and choose a non-zero section $s$ of $H^{0}(M, L)$. Now the foliation $\mathcal{F}(\omega), \omega \in H^{0}\left(M, \Omega^{1} \otimes L\right)$ is given by the meromorphic 1 -form $\frac{\omega}{s}$ in $M$. 
Definition 2.1 The meromorphic section $s \in H^{0}(M, L)$ is called the integrating factor of $\mathcal{F}(\omega), \omega \in H^{0}\left(M, \Omega^{1} \otimes L\right)$ if $\frac{\omega}{s}$ is a closed meromorphic function in $M . \mathcal{F}(\omega)$ is called integrable if there exists an integrating factor $s \in H^{0}(M, L)$ such that $\frac{\omega}{s}=d f$, where $f$ is a meromorphic function on $M$. In this case $f$ is constant on the leaves of $\mathcal{F}$. We also say that $f$ is a first integral of $\mathcal{F}$.

Let $\mathcal{F}$ be an integrable holomorphic foliation given by a without zero holomorphic 1-form $\omega \in H^{0}\left(M, \Omega^{1} \otimes L\right)$. Let also $f$ be a first integral of $\mathcal{F}$. The fibers of $f$ has the same linear bundle which we denote it by $L_{A}$, where $A$ is a generic fiber of $f$. Let $A_{1}, A_{2}, \ldots, A_{s}$ be irreducible components of the fibers of $f$ in which $f$ has multiplicities greater than one, namely $n_{1}, n_{2}, \ldots, n_{s}$, respectively. It is easy to see that

$$
L=L_{2 A-\sum_{1}^{s}\left(n_{i}-1\right) A_{i}}
$$

For the projective space $\mathbb{P}^{2}$ we have $\operatorname{Pic}\left(\mathbb{P}^{2}\right) \cong \mathbb{Z}$. We can take a line $H$ as the generator of $\operatorname{Pic}\left(\mathbb{P}^{2}\right)$. Therefore every line bundle in $\mathbb{P}^{2}$ is of the form $L_{d . H}$, where $d$ is an integer. It is easy to see that for a line bundle coming from a foliation $d$ is positive. In this case $d$ is called the degree of the foliation. Every degree $d$ holomorphic foliation in $\mathbb{P}^{2}$ is given by a polynomial 1-form $P d x-Q d y$ in the affine chart $\mathbb{C}^{2}=\mathbb{P}^{2}-H$.

\section{Picard-Lefschetz Theory}

This section is mainly based on the papers [La] and [Ho2]. For more information the reader is referred to those papers. Throughout the text when we do not write the coefficients used in the homology we mean homology with coefficients in $\mathbb{Z}$.

Let $f$ be a meromorphic function on a complex manifold $M$ of dimension two. The indeterminacy set $\mathcal{R}$ of $f$ contains the points of $M$ in which $f$ has the form $\frac{0}{0}$. $\mathcal{R}$ is a discrete set and the following holomorphic function is well-defined:

$$
f: M-\mathcal{R} \rightarrow \mathbb{P}^{1}
$$

We use the following notations

$$
L_{K}=f^{-1}(K), M_{K}=\overline{L_{K}}, K \subset \mathbb{P}^{1}
$$

For any point $c \in \mathbb{P}^{1}$ by $L_{c}$ and $M_{c}$ we mean the set $L_{\{c\}}$ and $M_{\{c\}}$, respectively. Throughout the text by a compact $f$-fiber we mean $M_{t}$ and by a $f$-fiber only we mean $L_{t}$. Performing a finite number of blow-ups (see [CaSa]) at the points of $\mathcal{R}$ and using Ehresmann's Fibration Theorem 
(see $[\mathrm{La}]$ ), we can see that there exists a finite subset $C=\left\{c_{1}, c_{2}, \ldots, c_{r}\right\}$ of $\mathbb{P}^{1}$ such that $f$ fibers $M-\mathcal{R}$ locally trivially over $B=\mathbb{P}^{1}-C$, i.e., for every point $b \in B$ there is a neighborhood $U$ of $b$ and a $C^{\infty}$-diffeomorphism $\phi: U \times f^{-1}(c) \rightarrow f^{-1}(U)$ such that $f \circ \phi=\pi_{1}=$ the first projection. We say that $C$ is the set of critical values of $f$. The regularity of $f$ along a fiber $L_{c}$ does not imply that it is a fiber bundle over a neighborhood of $c$. This situation happens when $M_{c}$ has a tangency point with the divisor of the blow-up in which the leaves separate from each other. In this case we say that the critical point related to the value $c$ is in $\mathcal{R}$.

Now we are able to use the Picard-Lefschetz theory for understanding the topology of the fibers of $f$. Let $\lambda$ be a path in $B$ with the initial and end points $b_{0}$ and $b_{1}$. There is an isotopy

$$
H: L_{b_{0}} \times[0,1] \rightarrow L_{\lambda}
$$

such that for all $x \in L_{b_{0}}, t \in[0,1]$

$$
H(x, 0)=x, H(x, t) \in L_{\lambda(t)}
$$

For every $t \in[0,1]$ the map $h_{t}=H(., t)$ is a homeomorphism between $L_{b_{0}}$ and $L_{\lambda(t)}$. The different choices of $H$ and paths homotopic to $\lambda$ in $B$ would give the class of isotopic maps

$$
\left\{h_{\lambda}: L_{b_{0}} \rightarrow L_{b_{1}}\right\}
$$

where $h_{\lambda}()=.H(., 1)$. The class $\left\{h_{\lambda}: L_{b_{0}} \rightarrow L_{b_{1}}\right\}$ defines the map

$$
h_{\lambda}: H_{1}\left(L_{b_{0}}\right) \rightarrow H_{1}\left(L_{b_{1}}\right)
$$

For any regular value $b$ of $f$, we can define

$$
\begin{gathered}
h: \pi_{1}(B, b) \times H_{1}\left(L_{b}\right) \rightarrow H_{1}\left(L_{b}\right) \\
h(\lambda, .)=h_{\lambda}(.)
\end{gathered}
$$

The action of $\pi_{1}(B, b)$ through $h$ on $H_{1}\left(L_{b}\right)$ is called the action of monodromy on the first homology group of $L_{b}$. We also say that $H_{1}\left(L_{c}\right)$ is a $\pi_{1}(B, b)$ module.

Let $q$ be an indeterminacy point of $f$. Let $\tau: \tilde{M} \rightarrow M$ be the map of successive blow-ups in $q$ such that $f \circ \tau$ is holomorphic in $\tau^{-1}(M, q)$ and there is an irreducible component $D_{1} \cong \mathbb{P}^{1}$ of the divisor $\tau^{-1}(q)$ such that $\left.f \circ \tau\right|_{D_{1}}$ is an isomorphism (see [CaSa] for details of such blow-ups). Every $\tau^{-1}\left(M_{t}\right)$ passes through $D_{1}$ transversally. Therefore we can choose a continuous family of small cycles in $\tau^{-1}\left(M_{t}\right)$ around $D_{1}$. The image of this 
family by $\tau$ is simply called a continuous family of cycles around $q$. This family is not defined for $t \in \mathbb{P}^{1}$ such that $\tau^{-1}\left(M_{t}\right)$ contains an irreducible component of $\tau^{-1}(q)$. Particularly it is defined for all $t \in \mathbb{P}^{1}-C$. In fact a $\delta_{t}$ is obtained by monodromy along an arbitrary path from a fixed $\delta_{b}$. The action of monodromy on $\delta_{b}$ is identity.

Definition 3.1 Let $K$ be a subset of $B$ and $b$ be a point in $K \backslash C$. Any relative 2-cycle of $L_{K}$ modulo $L_{b}$ is called a 2-thimble above $(K, b)$ and its boundary in $L_{b}$ is called a vanishing 1-cycle above $K$.

Suppose that $f$ has a non-degenerate critical point at $p_{i} \in M-\mathcal{R}$ and $f\left(p_{i}\right)=c_{i}$. Let $\lambda_{i}$ be a path in $B \cup\left\{c_{i}\right\}$ connecting a regular value $b$ to $c_{i}$. This path passes through $c_{i}$ only in its end point. The leaf $L_{b}$ contains a cycle which vanishes above $\lambda_{i}$ and called the Lefschetz vanishing cycle. Roughly speaking, when the value $x$ varies from $b$ to $c_{i}$, the Lefschetz vanishing cycle in $L_{x}$ moves in the leaves and arrives to the leaf $L_{c_{i}}$ as the point $p_{i}$. The locus of this movement is exactly the related 2-thimble.

Fix a point $\infty \in \mathbb{P}^{1}$, which may be a critical value. Let $C^{\prime}=\left\{c_{1}, c_{2}, \ldots, c_{r}\right\}$ be the subset of $\mathbb{C}=\mathbb{P}^{1}-\{\infty\}$ containing critical values of $f$.

Consider a system of $r$ paths $\lambda_{1}, \ldots, \lambda_{r}$ starting from $b$ and ending at $c_{1}, c_{2}, \ldots, c_{r}$, respectively, and such that:

1. each path $\lambda_{i}$ has no self intersection points ;

2. two distinct path $\lambda_{i}$ and $\lambda_{j}$ meet only at their common origin $\lambda_{i}(0)=$ $\lambda_{j}(0)=b$.

This system of paths is called a distinguished system of paths. Let $K$ be the union of these paths and small disks around $c_{i}$ 's. The set of vanishing cycles above $K$ in $L_{b}$ is called a distinguished set of vanishing cycles related to the critical points $c_{1}, c_{2}, \ldots, c_{r}$.

Theorem 3.1 ([Ho2, Theorem 2.2.1]) Suppose that $H_{1}\left(M-M_{\infty}, \mathbb{Q}\right)=0$. Then a distinguished set of vanishing 1-cycles related to the critical points in the set $C \backslash\{\infty\}=\left\{c_{1}, c_{2}, \ldots, c_{r}\right\}$ generates $H_{1}\left(L_{b}, \mathbb{Q}\right)$.

Again note that in the above theorem $\infty$ can be a critical value of $f$. If $M_{\infty}$ is smooth and its homology class in $H_{2}\left(M, \mathbb{Q}\right.$ ) is not zero (for example if $M_{\infty}$ is a smooth hyperplane section) then the condition $H_{1}(M, \mathbb{Q})=0$ implies that $H_{1}\left(M-M_{\infty}, \mathbb{Q}\right)=0$. The reason is as follows: By Leray (or Thom-Gysin) isomorphism ( see [Ch, p. 537]) we have $H_{2}\left(M, M-M_{\infty}\right) \simeq H_{0}\left(M_{\infty}\right) \simeq \mathbb{Z}$. Now we write the long exact sequence of the pair $\left(M, M-M_{\infty}\right)$

$$
\cdots \rightarrow H_{2}\left(M-M_{\infty}\right) \stackrel{i}{\rightarrow} H_{2}(M) \rightarrow \mathbb{Z} \stackrel{j}{\rightarrow} H_{1}\left(M-M_{\infty}\right) \rightarrow 0
$$


Therefore $H_{1}\left(M-M_{\infty}\right)=\mathbb{Z} / n \mathbb{Z}$ or $\mathbb{Z}$ for some natural number $n$. In the first case we have $H_{1}\left(M-M_{\infty}, \mathbb{Q}\right)=0$. In the second case $j$ is one to one and so $i$ is surjective. But this means that the intersection of $M_{\infty}$ with any 2-cycle in $H_{2}(M)$ is zero. By Poincaré duality the class of $M_{\infty}$ in $H_{2}(M, \mathbb{Q})$ must be zero which is a contradiction.

Definition 3.2 The cycle $\delta$ in a regular fiber $L_{b}$ is called simple if the action of $\pi_{1}(B, b)$ on $\delta$ generates $H_{1}\left(L_{b}, \mathbb{Q}\right)$.

Note that in the above definition we have considered the homology group with rational coefficients. Of course, not all cycles are simple. For instance if the meromorphic function in a local coordinate $(x, y)$ around $q \in \mathcal{R}$ has the form $\frac{x}{y}$, then the cycle around $q$ in each leaf has this property that it is fixed under the action of monodromy, therefore it cannot be simple. In the next paragraph we are going to introduce some fibrations with simple cycles.

Lefschetz pencil: The hyperplanes of $\mathbb{P}^{n}$ are points of the dual projective space $\check{\mathbb{P}}^{n}$. We use the following notation:

$$
H_{y} \subset \mathbb{P}^{n}, y \in \check{\mathbb{P}}^{n}
$$

Let $M$ be a closed irreducible smooth subvariety of $\mathbb{P}^{n}$. Its dual variety $\check{M}$ consists of all points $y$ in $\check{\mathbb{P}}^{n}$ such that $H_{y}$ is tangent to $M$ at some point. $\check{M}$ may have singularities. A Lefschetz pencil in $\mathbb{P}^{n}$ consists of all hyperplanes which contain a fixed $(n-2)$-dimensional projective space $A$, which is called the axis of the pencil. We denote a pencil by

$$
\left\{H_{t}\right\}_{t \in G}
$$

or $G$ itself, where $G$ is a projective line in $\check{\mathbb{P}}^{n}$. The pencil $\left\{H_{t}\right\}_{t \in G}$ is in general position with respect to $M$ if $G$ is in general position with respect to $\check{M}$. Sometimes we parameterize the line $G \cong \mathbb{P}^{1}$ and consider the meromorphic function $f$ on $M$ induced by the pencil. The meromorphic function associated to a pencil in general position is called the generic Lefschetz meromorphic function of the pencil and has the following properties:

1. In a local coordinates system $\left(x_{1}, x_{2}, \ldots, x_{n}\right)$ around an indeterminacy point $q$ of $f, f$ has the form $\frac{x_{1}}{x_{2}}$;

2. $f$ has $r=\operatorname{deg}(\check{M})$ non-degenerate critical points $p_{1}, \ldots, p_{r}$ in $M-A$ such that $f\left(p_{i}\right)=c_{i}$ 's are distinct values in $\mathbb{P}^{1}$;

3. For every critical value $c_{i}$ of $f$, the hyperplane $H_{c_{i}}$ has a unique tangency of order two with $M$ which lies out of $A$. The other hyperplanes are transverse to $M$ (see [La]). 
Theorem 3.2 Suppose that $H_{1}\left(M-M_{\infty}, \mathbb{Q}\right)=0$ and $H_{1}\left(M_{\infty}\right) \neq 0$, where $M_{\infty}$ is a regular fiber of a generic Lefschetz meromorphic function $f$. Then every Lefschetz vanishing cycle in a regular fiber of $f$ is simple.

Note that the regular compact fibers of $f$ have the same topology and so $H_{1}\left(M_{\infty}\right) \neq 0$ means that the genus of any regular compact fiber is greater than 0 and so the fibration is not rational.

Almost all the arguments to prove the above theorem exist in [La]. That article has worked with the topology of $M_{t}$ 's, and not $L_{t}$ 's. Therefore we have proved above theorem in [Ho2, Theorem 2.3.2].

Let $\mathcal{F}$ be a foliation in $\mathbb{P}^{2}$ with a first integral of the type $\frac{F^{p}}{G^{q}}$, where $F$ and $G$ are two relatively prime irreducible polynomials in an affine chart $\mathbb{C}^{2}$ of $\mathbb{P}^{2}, \frac{\operatorname{deg}(F)}{\operatorname{deg}(G)}=\frac{q}{p}$ and $\operatorname{gcd}(p, q)=1$. Assume that $\mathcal{F}$ has the following generic properties:

1. $\{F=0\}$ and $\{G=0\}$ are smooth and intersect each other transversally;

2. The critical points of $\frac{F^{p}}{G^{q}}$ in $\mathbb{P}^{2} \backslash(\{F=0\} \cup\{G=0\})$ are non-degenerate with distinct images.

Let $c_{1}, c_{2}, \ldots, c_{r}$ denote these critical values. In [Ho2] it is proved that these conditions are generic, i.e. there exists a dense open subsets in the space of coefficients of $F$ and $G$ such that all polynomials $F$ and $G$ whose coefficients are chosen from this set, satisfy the conditions 1 and 2 . The meromorphic function $\frac{F^{p}}{G^{q}}$ is a fiber bundle over $\mathbb{P}^{1}-\left\{c_{1}, c_{2}, \ldots, c_{r}, 0, \infty\right\}$. It has multiplicity $p$ along $F=0$ and $q$ along $G=0$.

Theorem 3.3 ([Ho2]) If $\operatorname{deg}(F)+\operatorname{deg}(G)>4$ then every Lefschetz vanishing cycle in a regular fiber of $\frac{F^{p}}{G^{q}}$ is simple.

It is easy to see that $\frac{F^{p}}{G^{q}}$ is a rational fibration (a fibration whose generic fiber has genus zero) if and only if $\operatorname{deg}(F)+\operatorname{deg}(G) \leq 2$. In fact only in the case $\operatorname{deg}(F)+\operatorname{deg}(G) \leq 2$ the fibration $\mathcal{F}$ has reducible critical fibers. In the case $\operatorname{deg}(F)=\operatorname{deg}(G)=1$, Theorem 3.3 is not true. In this case it is easy to find counterexamples for the forthcoming Theorem 6.2 by performing deformations inside the logarithmic foliations.

If $p=q=1$ then $\frac{F^{p}}{G^{q}}$ is a Lefschetz meromorphic function and the above theorem is a particular case of Theorem 3.2. In the case where $G$ is a linear polynomial we have $p=1$ and $q=\operatorname{deg}(F)$. Considering $G=0$ as the line at infinity in $\mathbb{P}^{2}$, we have the Hamiltonian fibrations in $\mathbb{C}^{2}$. In this case the above theorem is proved by Ilyashenko [Il] using a theorem of Zizcenko. 


\section{Deformation of Holomorphic Foliations and Abelian Integrals}

Let $\mathcal{F}$ be an integrable holomorphic foliation in $\mathcal{F}(M, L)$ and $\mathcal{F}_{\epsilon}\left(\omega_{\epsilon}\right) \in$ $\mathcal{F}(M, L), \epsilon \in(\mathbb{C}, 0)$ a holomorphic deformation of $\mathcal{F}=\mathcal{F}_{0}$. The set $H^{0}\left(M, \Omega^{1} \otimes L\right)$ is a vector space and so we can write

$$
\omega_{\epsilon}=\omega_{0}+\epsilon \omega_{1}+\epsilon^{2} \omega_{2}+\cdots, \quad \omega_{i} \in H^{0}\left(M, \Omega^{1} \otimes L\right)
$$

The 1-form $\omega_{1}$ is called the tangent vector of the deformation.

Let $\delta$ be a cycle in a leaf of $\mathcal{F}$ and $\Sigma \simeq(\mathbb{C}, 0)$ a holomorphic section to $\mathcal{F}$ in a point $p \in \delta$. Let also $s$ be an integrating factor of $\omega_{0}$ whose zero divisor does not intersect $\delta$ and

$$
\frac{\omega_{0}}{s}=d f
$$

where $f$ is a meromorphic function on $M$.

Throughout the text we assume that the transverse section $\Sigma$ is parameterized by $t=\left.f\right|_{\Sigma}$. Assume that the holonomy of $\mathcal{F}$ along $\delta$ is identity. Note that if $f$ has multiplicity one along the leaf containing $\delta$ then the holonomy is always identity. We can consider the collection of $\mathcal{F}_{\epsilon}$ 's as a codimension two foliation $\overline{\mathcal{F}}=\left\{\mathcal{F}_{\epsilon}\right\}_{\epsilon \in(\mathbb{C}, 0)}$ in $M \times(\mathbb{C}, 0)$ and $\Sigma \times(\mathbb{C}, 0)$ as a transverse section to $\overline{\mathcal{F}}$. So we have the holonomy map defined by

$$
\begin{gathered}
H: \Sigma \times(\mathbb{C}, 0) \rightarrow \Sigma \times(\mathbb{C}, 0) \\
H(t, \epsilon)=\left(h_{\epsilon}(t), \epsilon\right)
\end{gathered}
$$

$h_{\epsilon}(t)$ is a holomorphic function in $\epsilon$ and $t$ and is called the holonomy of $\mathcal{F}_{\epsilon}$ along the path $\delta$ (Note that by hypothesis $h_{0}(t) \equiv t$ ). We write

$$
h_{\epsilon}(t)-t=M_{1}(t) \epsilon+M_{2}(t) \epsilon^{2}+\cdots+M_{i}(t) \epsilon^{i}+\cdots, i ! . M_{i}(t)=\left.\frac{\partial^{i} h_{\epsilon}}{\partial \epsilon^{i}}\right|_{\epsilon=0}
$$

$M_{i}$ is called the $i$-th Melnikov function of the deformation along the path $\delta$. Let $M_{1} \equiv M_{2} \equiv \cdots \equiv M_{k-1} \equiv 0$ and $M_{k} \not \equiv 0$. It is a well known fact that the multiplicity of $M_{k}$ at $t=0$ is the number of limit cycles (more precisely the number of fixed points of the holonomy $h_{\epsilon}$ ) which appears around $\delta$ after the deformation. This fact shows the importance of these functions in the local study of Hilbert 16-th problem. The following proposition gives us a nice formula for the first Melnikov function.

Proposition 4.1 The first Melnikov function is given by

$$
M_{1}(t)=-\int_{\delta_{t}} \frac{\omega_{1}}{s}
$$

where $\omega_{1}$ is the tangent vector of the deformation and $\delta_{t}$ is the lifting up of $\delta$ in the leaf through $t \in \Sigma$. 
Proof: The proof is completely formal in the literature of differential equations (see $[\mathrm{Ro}]$ and $[\mathrm{Fr}]$ ). The deformed foliation is given by the meromorphic 1 -form

$$
d f+\epsilon \frac{\omega_{1}}{s}+O\left(\epsilon^{2}\right)
$$

Let $\delta_{t, h_{\epsilon}(t)}$ be a path in the leaf of $\mathcal{F}_{\epsilon}$ through $t$ which connects $t$ to $h_{\epsilon}(t)$ along the path $\delta$. Since $\Sigma$ is parameterized by $t=\left.f\right|_{\Sigma}$, by integrating the 1-form (4.1) over the path $\delta_{t, h_{\epsilon}(t)}$ we have

$$
h_{\epsilon}(t)-t+\epsilon\left(\int_{\delta_{t}} \frac{\omega_{1}}{s}+O(\epsilon)\right)+O\left(\epsilon^{2}\right)=0
$$

The coefficient of $\epsilon$ in the above equality gives us the desired equality.

We want to have an explicit formula for higher Melnikov functions. For this purpose we must classify a certain class of relatively exact 1-forms modulo an integrable foliation.

\section{Relatively Exact 1-forms}

First let us give the definition of a relatively exact 1-forms modulo a foliation.

Definition 5.1 Let $\mathcal{F}$ be a foliation in $M$. A meromorphic 1-form $\omega_{1}$ on $M$ is called relatively exact modulo $\mathcal{F}$ if the restriction of $\omega_{1}$ to each leaf $\mathcal{L}$ of $\mathcal{F}$ is exact, i.e., there is a meromorphic function $f$ on $\mathcal{L}$ such that $\left.\omega_{1}\right|_{L}=d f$.

In fact we are interested in the above definition when $\mathcal{F}$ is integrable. It is easy to check that a meromorphic 1 -form $\omega_{1}$ is relatively exact modulo $\mathcal{F}$ if and only if

$$
\int_{\delta} \omega_{1}=0
$$

for all closed cycles in the leaves of $\mathcal{F}$, where this integral is well-defined.

Relatively exact 1-forms have been studied by many authors. Ilyashenko in [Il] proves that if the integral of a polynomial 1-form of degree $n$ along a continuous family of level lines of a Morse type polynomial of two variables and of degree $n$ is identically equal to zero, then the form is relatively exact modulo the polynomial and then he proves that it must be exact. This is generalized to higher dimensions in $[\mathrm{Pu}]$. The classification of relatively exact polynomial 1-forms modulo an arbitrary polynomial is done by P. Bonnet in $[\mathrm{Bo}]$ and L. Gavrilov in [Ga]. J. Muciño in $[\mathrm{Mu}]$ has classified 
a certain class of relatively exact 1 -forms modulo a Lefschetz pencil. In a generalization of Ilyashenko's result to integrable foliations in $M$, I had to classify another types of relatively exact 1-forms in [Ho1].

Let $S$ be a complex curve. The meromorphic function $f: M \rightarrow S$ is called non-composite if a general compact $f$-fiber is irreducible. It is easy to see that $f: M \rightarrow S$ is non-composite if and only if $f$ cannot be factored as a composite

$$
M \stackrel{f^{\prime}}{\rightarrow} S^{\prime} \stackrel{i}{\rightarrow} S
$$

where $S^{\prime}$ is a complex curve and $i$ is a holomorphic map of degree greater than one.

Let $\mathcal{F}$ be an integrable foliation. There exists a complex curve $S$ and a non-composite meromorphic function $f: M \rightarrow S$ such that $f$ is constant in the leaves of $\mathcal{F}$ (see [Go]). We say that $f$ is a non-composite first integral of $\mathcal{F}$.

Let $f: M \rightarrow S$ be non-composite as above. Any other first integral $f^{\prime}: M \rightarrow S^{\prime}$ of $\mathcal{F}$ is factored as

$$
M \stackrel{f}{\rightarrow} S \stackrel{i}{\rightarrow} S^{\prime}
$$

where $i$ is a holomorphic function.

Poincaré in his article $[\mathrm{Po}]$ has studied integrable foliations in $\mathbb{P}^{2}$ and has proved (p. 52-53) that every integrable foliation $\mathcal{F}$ in $\mathbb{P}^{2}$ has a non-composite first integral $M \rightarrow \mathbb{P}^{1}$ (this is also a consequence of Stein factorization theorem).

Let $\mathcal{F}$ be an integrable foliation with the non-composite first integral $f: M \rightarrow S$ and $\omega_{1}$ a meromorphic 1-form in $M$ with the pole divisor

$$
D=\sum_{i=1}^{n} n_{i} D_{i}
$$

In what follows when we say that a meromorphic object (function, 1-form...) $Z$ has the pole divisor $D$, we mean that

$$
\operatorname{pol}(Z)+D \geq 0
$$

Let

$$
D_{0}=\sum_{i=1}^{n} D_{i}
$$

be the reduced part of $D$. 
Theorem 5.1 Keeping the notations used above, assume that

1. All $f$-fibers are connected (for us a $f$-fiber does not contain the indeterminacy points of $f$ );

2. $D$ is $\mathcal{F}$-invariant;

3. Every component of $D$ is an irreducible $f$-fiber ( $f$ may have multiplicity along some $D_{i}$ );

4. There exists a non $\mathcal{F}$-invariant Riemann surface embedded in $M-\mathcal{R}$.

Then every relatively exact meromorphic 1 -form $\omega_{1}$ modulo $\mathcal{F}$ with the pole divisor $D$ has the form

$$
\omega_{1}=d g+\omega
$$

where $g$ is a meromorphic function on $M$ with the pole divisor $D$ and $\omega$ is a meromorphic 1-form in $M$ inducing the foliation $\mathcal{F}$ and with the pole divisor $D+D_{0}$.

The above theorem is no more true if we assume that some $f$-fibers are disconnected. P. Bonnet in $[\mathrm{Bo}]$ gives the example $f=x(1+x y)$ in $\mathbb{C}^{2}$ having the disconnected fiber $f=0$. The 1 -forms $y^{k+1} d x+x y^{k} d y, k>0$ are relatively exact modulo $f$ but they are not of the form (5.3). Without the hypothesis of connectedness of $f$-fibers the classification of relatively exact polynomial 1-forms modulo a polynomial is done in [Bo]. In [Ho1] we have classified relatively exact 1 -form in $\mathbb{P}^{2}$ with non-invariant divisors. The forth condition is trivial for an algebraic manifold with a Lefschetz pencil in it. I do not know yet that the third and forth conditions in the above theorem is really necessary or not. The above classification of relatively exact 1-form will suffice to our purpose.

Proof: Let $\mathcal{R}$ be the indeterminacy set of $f$ and $C$ a non $\mathcal{F}$-invariant Riemann surface in $U=M-\mathcal{R}$. For any point $x \in U$ let

$$
L_{x} \cap C=\left\{p_{1}, p_{2}, \ldots, p_{e}\right\}
$$

where $L_{x}=f^{-1}(g(x))$ is the fiber through $x$ and $e$ is the intersection number of $C$ with a generic $f$-fiber ( $p_{i}$ 's are counted with multiplicity). Define

$$
\begin{gathered}
g: M \backslash\left(\cup_{i=1}^{n} D_{i}\right) \rightarrow \mathbb{C} \\
g(x)=\frac{1}{e}\left(\sum \int_{x}^{p_{i}} \omega_{1}\right)
\end{gathered}
$$

where $\int_{x}^{p_{i}}$ is an integral over a path in $L_{x}$ which connects $x$ to $p_{i}$. 
Since the $f$-fibers are connected, $\int_{x}^{p_{i}}$ is well-defined. The idea of the definition of $g$ comes from the paper [Mu]. According to the hypothesis this integral does not depend on the choice of the path connecting $x$ to $p_{i}$ on $L_{x}$. The function $g$ is a well-defined holomorphic function in $M \backslash\left(\cup_{i=1}^{n} D_{i}\right)$. We claim that $g$ is a meromorphic function on $M$ with pole divisor $D$. According to Levi extension theorem it is enough to prove that $g$ is meromorphic in $U$.

For instance let us prove that $g$ has a pole of order at most $n_{i}$ at $U \cap D_{i}$. Let $m_{i}$ be the multiplicity of $f$ along $D_{i}, c_{i}=f\left(D_{i}\right)$ be the value associated to $D_{i}$ and $x \in U \cap D_{i}$. In a small neighborhood of the path connecting $x$ to $p_{i}$ the function $\left(f-c_{i}\right)^{\frac{n_{i}}{m_{i}}}$ is a univalued holomorphic function and

$$
\int_{x}^{p_{i}} \omega_{1}=\left(f-c_{i}\right)^{-\frac{n_{i}}{m_{i}}} \int_{x}^{p_{i}}\left(f-c_{i}\right)^{\frac{n_{i}}{m_{i}}} \omega_{1}
$$

$\left(f-c_{i}\right)^{\frac{n_{i}}{m_{i}}} \omega_{1}$ is a holomorphic 1-form along $U \cap D_{i}$ and therefore integrals of this type has a pole of order at most $n_{i}$ at $\left\{D_{i}=0\right\}$. This implies that $g$ has pole of order at most $n_{i}$ at $\left\{D_{i}=0\right\}$. Note that in the above formulas we have chosen a local chart $z(c), c \in\left(S, c_{i}\right)$ around $c_{i}$ and instead of $z \circ f-z\left(c_{i}\right)$ we have simply written $f-c_{i}$.

Every integral $\int_{x}^{p_{i}} \omega_{1}$ satisfies the equation

$$
d\left(\int_{x}^{p_{i}} \omega_{1}\right) \wedge \omega_{0}=\omega_{1} \wedge \omega_{0}
$$

where $\omega_{0}$ is a meromorphic 1 -form inducing $\mathcal{F}$. The function $g$ is the mean value of these integrals and so

$$
\begin{gathered}
d g \wedge \omega_{0}=\omega_{1} \wedge \omega_{0} \Rightarrow\left(\omega_{1}-d g\right) \wedge \omega_{0}=0 \\
\Rightarrow \omega_{1}=d g+\omega
\end{gathered}
$$

where $\omega$ is a meromorphic 1 -form inducing $\mathcal{F}$ and with the pole divisor $D+D_{0}$.

Let $\mathcal{F}$ be a holomorphic foliation in $M$ considered in Theorem 5.1. Assume that $\mathcal{F}$ has a non composite meromorphic first integral $f\left(S=\mathbb{P}^{1}\right)$. Denote by $D$ a generic fiber of $f$.

Corollary 5.1 Every relatively exact 1-form $\omega_{1}$ in $M$ with the pole divisor $n D$ has the form

$$
\omega_{1}=d g+p d f
$$

where $g$ and $p$ are meromorphic functions with the pole divisors $n D$ and $(n-1) D$, respectively. 


\section{Foliations with a center in $\mathbb{P}^{2}$}

Let $\mathcal{F}$ be a germ of singular foliation at $\left(\mathbb{C}^{2}, 0\right)$. We say that $0 \in \mathbb{C}^{2}$ is a center singularity of $\mathcal{F}$ or simply a center of $\mathcal{F}$, if there exists a germ of holomorphic function $f:\left(\mathbb{C}^{2}, 0\right) \rightarrow(\mathbb{C}, 0)$ which has non-degenerate critical point at $0 \in \mathbb{C}^{2}$, and the leaves of $\mathcal{F}$ near 0 are given by $f=$ const.. The point 0 is also called a Morse singularity of $f$. Morse lemma in the complex case implies that there exists a local coordinate system $(x, y)$ in $\left(\mathbb{C}^{2}, 0\right)$ with $x(0)=0, y(0)=0$ and such that $f(x, y)=x^{2}+y^{2}$. Near the center the leaves of $\mathcal{F}$ are homeomorphic to a cylinder, therefore each leaf has a nontrivial closed cycle that will be called the Lefschetz vanishing cycle.

Let $\mathcal{F}(d)$ be the space of degree $d$ holomorphic foliations in $\mathbb{P}^{2}$ and $\mathcal{M}(d)$ the closure of the set of foliations of degree $d$ and with at least one center in $\mathcal{F}(d)$. The following example gives us a huge number of these foliations: Let $\tau: \mathbb{P}^{2} \rightarrow M$ be a holomorphic map between $\mathbb{P}^{2}$ and a complex compact manifold $M$ with $\operatorname{dim}(M) \geq 3$. Let also $\mathcal{F}$ be a holomorphic codimension one singular foliation in $M$ (see [Li]). We say that $\tau$ has a tangency point $a \in \mathbb{P}^{2}$ of order two with $\mathcal{F}$ if $\tau(a)$ is a regular point of the foliation $\mathcal{F}, \mathcal{F}$ in a coordinate $(x, y) \in\left(\mathbb{C}^{n-1}, 0\right) \times(\mathbb{C}, 0)$ around $a$ is given by $y=$ const. and $a$ is a non-degenerate critical point of $y \circ \tau$. This says that the pullback foliation $\tau^{*}(\mathcal{F})$ has a center at the point $a$.

I have learned the statement and proof of the following proposition from A. Lins Neto.

Proposition 6.1 $\mathcal{M}(d)$ is an algebraic subset of $\mathcal{F}(d)$.

The proof of this proposition is given in the end of this section. Let $\mathcal{P}_{d}$ be the set of polynomials of maximum degree $d$ in $\mathbb{C}^{2}$ and

$$
(F, G) \in \mathcal{P}_{a+1} \times \mathcal{P}_{b+1}, \frac{a+1}{b+1}=\frac{q}{p}, \quad \operatorname{gcd}(p, q)=1
$$

The foliation $\mathcal{F}=\mathcal{F}(p G d F-q F d G)$ has the first integral:

$$
f: \mathbb{P}^{2} \backslash(\{F=0\} \cap\{G=0\}) \rightarrow \mathcal{S}, f(x, y)=\frac{F(x, y)^{p}}{G(x, y)^{q}}
$$

i.e., the leaves of the foliation $\mathcal{F}$ are contained in the level surfaces of $f$. Let $\mathcal{I}(a, b)$ be the closure of the set of the mentioned holomorphic foliations in $\mathcal{F}(d)$. Our main result in this section is the following:

Theorem 6.1 $\mathcal{I}(a, b), a+b>2$ is an irreducible component of $\mathcal{M}(d)$, where $d=a+b$. 
This result is announced in [Ho1]. We can restate our main theorem as follows: Let $\mathcal{F} \in \mathcal{I}(a, b), p$ one of the center singularities of $\mathcal{F}$ and $\mathcal{F}_{t}$ a holomorphic deformation of $\mathcal{F}$ in $\mathcal{F}(d)$, where $d=a+b$, such that its unique singularity $p_{t}$ near $p$ is still a center.

Theorem 6.2 In the above situation, if $a+b>2$ then there exists an open dense subset $U$ of $\mathcal{I}(a, b)$, such that for all $\mathcal{F}(p G d F-q F d G) \in U, \mathcal{F}_{t}$ admits a meromorphic first integral. More precisely, there exist polynomials $F_{t}$ and $G_{t}$ such that $\mathcal{F}_{t}=\mathcal{F}\left(p G_{t} d F_{t}-q F_{t} d G_{t}\right)$, where $F_{t}$ and $G_{t}$ are holomorphic in $t$ and $F_{0}=F$ and $G_{0}=G$.

This theorem also says that the persistence of one center implies the persistence of all other centers and dicritical singularities (the points of $\{F=$ $0\} \cap\{G=0\})$.

The classification of degree two polynomial differential equations was done by Dulac in [Du]. Going to the language of holomorphic foliations in $\mathbb{P}^{2}$, instead of using the language of polynomial differential equations, this classification was completed in [CeLi] for degree two holomorphic foliation in $\mathbb{P}^{2}$. This classification for some degree three differential equations is done in [Ll]. Deformation of real Hamiltonian equations with a center singularity, generating limit cycles from the Lefschetz vanishing cycles of the center, has been one of the methods of approach to Hilbert sixteen problem, for this see Roussarie's book [Ro] and its reference. Yu. S. Ilyashenko in [Il] shows that the persistence of a center after deformation of a generic Hamiltonian equation implies that the deformed equation is also Hamiltonian. He uses this fact to get a certain number of limit cycles after deformation of Hamiltonian equations. Theorem 6.1 is a generalization of the Ilyashenko's result. The case $p=q=1$ was studied by J. Muciño in $[\mathrm{Mu}]$. With more strong hypotheses on the tangent vector of the deformation, i.e. the vector $\omega_{1}$ in $\mathcal{F}_{t}\left(d f+t \omega_{1}+\right.$ h.o.t. $)$, he succeeded in proving that $\omega_{1}$ is a relatively exact 1 -form and then classifies them. This result, however, does not imply that the persistence of just one center implies the persistence of all others.

Proof of Theorem 6.1: Since $\mathcal{I}(a, b)$ is parameterized by $\mathcal{P}_{a+1} \times \mathcal{P}_{b+1}$, $\mathcal{I}(a, b)$ is an irreducible variety. Let $\mathcal{I}_{m}(a, b)$ be the subset of $\mathcal{I}(a, b)$ containing $\mathcal{F}(p G d F-q F d G)$ with the properties 1,2 before Theorem 3.3. $\mathcal{I}_{m}(a, b)$ is an open dense subset of $\mathcal{I}(a, b)$ (see [Ho2]. Let $\mathcal{F}(p G d F-q F d G) \in \mathcal{I}_{m}(a, b)$ and $\mathcal{F}_{\epsilon}\left(p G d F-q F d G+\epsilon \omega_{1}+\right.$ h.o.t. $) \in \mathcal{F}(d)$ a deformation of $\mathcal{F}$ such that there is $\mathcal{F}_{\epsilon_{i}}, i=1,2 \ldots$ with this property that $\mathcal{F}_{\epsilon_{i}}$ has a center $p_{i}$ near a fixed center $p$ of $\mathcal{F}$. Since $\mathcal{F}_{\epsilon}$ is of degree $d, \frac{\omega}{F G}$ is a meromorphic 1-form in $\mathbb{P}^{2}$ with poles only in $\{F=0\}$ and $\{G=0\}$ (there is no pole along the line at infinity). The first Melnikov function of this deformation along a 
Lefschetz vanishing cycle around $p$ is zero. Therefore

$$
\int_{\delta} \frac{\omega_{1}}{F G}=0
$$

for all vanishing cycles $\delta$ in the leaves around $p$ and by Theorem 3.3 we conclude that the equality (6.1) holds for all closed cycles $\delta$ in the leaves of the foliation $\mathcal{F}$, where the integral is defined. Partially we obtain that the residue of $\frac{\omega_{1}}{F G}$ on a leaf around any $q \in\{F=0\} \cap\{G=0\}$ is zero. Therefore the 1 -form $\frac{\omega_{1}}{F G}$ is relatively exact modulo the foliation $\mathcal{F}(p G d F-q F d G)$. By Theorem 5.1, there exist polynomials $B$ and $R$ of degree at most $d+2$ such that

$$
\begin{aligned}
& \frac{\omega_{1}}{F G}=d\left(\frac{B}{F G}\right)-\frac{R}{F G}\left(\frac{p G d F-q F d G}{F G}\right) \Longrightarrow \\
& \omega_{1}=\frac{F G d B-B d(F G)-R(p G d F-q F d G)}{F G}
\end{aligned}
$$

This implies that

$$
\begin{gathered}
F|B+p R, G| B-q R \Longrightarrow \\
B+p R=-(p+q) F Q, B-q R=(p+q) G P \Longrightarrow \\
B=p G P-q F Q, R=-G P-F Q
\end{gathered}
$$

where $P$ and $Q$ are two polynomials of degree at most $\operatorname{deg}(F)$ and $\operatorname{deg}(G)$, respectively. Substituting this in (6.2) we have

$$
\omega_{1}=p G d P-q P d G+p Q d F-q F d Q
$$

Until now we have proved that

$$
\begin{gathered}
{\left[\omega_{1}\right] \in T_{\mathcal{F}} \mathcal{M}(d) \Longrightarrow} \\
\omega_{1}=p G d P-q P d G+p Q d F-q F d Q,(P, Q) \in \mathcal{P}_{a+1} \times \mathcal{P}_{b+1} \Longrightarrow \\
{\left[\omega_{1}\right] \in T_{\mathcal{F}} \mathcal{I}(a, b)}
\end{gathered}
$$

this and the fact that $\mathcal{I}(a, b) \subset \mathcal{M}(d)$ imply that

$$
T_{\mathcal{F}} \mathcal{M}(d)=T_{\mathcal{F}} \mathcal{I}(a, b), \forall \mathcal{F} \in \mathcal{I}_{m}(a, b)
$$

Since $\mathcal{I}_{m}(a, b)$ is an open dense subset of $\mathcal{I}(a, b)$, we conclude that $\mathcal{I}(a, b)$ is an irreducible component of $\mathcal{M}(d)$.

In the language of differential equations (see [Ro]) what we have proved in Theorem 6.1 is the following: The Bautin variety (zeros of Bautin ideal) of a Lefschetz vanishing cycle of a generic $\mathcal{F} \in \mathcal{I}(a, b)$ with $\mathcal{F}(d), d=a+b$, as the deformation space, is $\mathcal{I}(a, b)$. Note that the Bautin variety is defined locally but $\mathcal{I}(a, b)$ is a closed algebraic set in $\mathcal{F}(M, L)$. 
Let $X$ be an irreducible component of $\mathcal{M}(d), \mathcal{F}\left(\omega_{0}\right) \in X$ and $p$ be a center of $\mathcal{F}$. There is a coordinate $(x, y)$ in a small neighborhood $U$ of $p$ such that in this coordinate $p=(0,0)$ and

$$
\omega_{0}=g d(\tilde{f}), \tilde{f}=\frac{1}{2}\left(x^{2}+y^{2}\right)+\text { h.o.t., } g(0) \neq 0
$$

where $\tilde{f}$ and $g$ are holomorphic functions on $U$. Define $T_{\mathcal{F}}^{*} X$ as the set of all 1-forms $\left[\omega_{1}\right] \in T_{\mathcal{F}} \mathcal{F}(M, L)$ such that

$$
\int_{\delta} \frac{\omega_{1}}{g}=0
$$

for all Lefschetz vanishing cycles in the leaves of $\mathcal{F}$ around $p$. By using the formula of first Melnikov function we know that

$$
T_{\mathcal{F}} X \subset T_{\mathcal{F}}^{*} X
$$

$X$ is called a good irreducible component of $\mathcal{M}(d)$ if for a generic choice of $\mathcal{F} \in X$, the equality holds in (6.4).

The above definition does not depend on the choice of $g$. More precisely, if

$$
\omega_{0}=g d \tilde{f}=g^{\prime} d \tilde{f}^{\prime} \Rightarrow \frac{g}{g^{\prime}} d \tilde{f}=d \tilde{f}^{\prime} \Rightarrow d\left(\frac{g}{g^{\prime}}\right) \wedge \omega_{0}=0
$$

This means that $\frac{g}{g^{\prime}}$ is constant on the leaves of $(\mathcal{F}, p)$ and so

$$
\int_{\delta} \frac{\omega_{1}}{g^{\prime}}=\int_{\delta} \frac{g}{g^{\prime}} \frac{\omega_{1}}{g}=\frac{g}{g^{\prime}} \int_{\delta} \frac{\omega_{1}}{g^{\prime}}=0
$$

which implies that the definition does not depend on $g$.

Proposition 6.2 $\mathcal{I}(a, b)$ is a good irreducible component of $\mathcal{M}(d)$.

Proof: The proof is the same as the proof of Theorem 6.1.

Challenge: We have proved that for $d>2$

$$
\mathcal{I}(0, d), \mathcal{I}(1, d-1), \mathcal{I}(2, d-2), \ldots, \mathcal{I}\left(\left[\frac{d}{2}\right], d-\left[\frac{d}{2}\right]\right)
$$

are good irreducible components of $\mathcal{M}(d)$ (Note that $\mathcal{I}(a, b)=\mathcal{I}(b, a)$ ). Which are the other irreducible components of $\mathcal{M}(d)$ ? Is any other irreducible component of $M$ good? For the cases $d=1,2$ the complete description of the irreducible components of $\mathcal{M}(d)$ is done in [Du] and [CeLi]. Let us introduce an algebraic set in $\mathcal{F}(M, L)$ which seems to be an irreducible component of $\mathcal{M}(d)$ : 
Let $\mathcal{F}$ be a foliation in $\mathbb{P}^{2}$ given by the polynomial 1 -form

$$
\omega(f, \lambda)=\omega\left(f_{1}, \ldots, f_{r}, \lambda_{1}, \ldots, \lambda_{r}\right)=f_{1} \cdots f_{r} \sum_{i=1}^{r} \lambda_{i} \frac{d f_{i}}{f_{i}}
$$

where the $f_{i}$ 's are irreducible polynomials in $\mathbb{C}^{2}, \sum d_{i} \lambda_{i}=0$ and $d_{i}=$ $\operatorname{deg}\left(f_{i}\right) . \mathcal{F}$ is called a logarithmic foliation and it has the multi-valued first integral $f=f_{1}^{\lambda_{1}} \cdots f_{r}^{\lambda_{r}}$ in $U=\mathbb{P}^{2} \backslash\left(\cup_{i=1}^{r}\left\{f_{i}=0\right\}\right)$. We can prove that generically, the degree of $\mathcal{F}$ is $d=\sum_{i=1}^{r} d_{i}-2$ and has $d^{2}+d+1-\sum_{1 \leq i<j \leq r} d_{i} d_{j}$ centers.

Let $\mathcal{L}\left(d_{1}, d_{2}, \ldots, d_{r}\right)$ be the set of all logarithmic foliations of the above type. Is $\mathcal{L}\left(d_{1}, d_{2}, \ldots, d_{r}\right)$ an (a good) irreducible component of $\mathcal{M}(d)$, where $d=\sum_{i=1}^{r} d_{i}-2$ ? As far as I know, there is no theorem similar to Theorem 3.3 for logarithmic foliations.

Recently some developments in the above problem is made by the author of these lines in [Ho4] for logarithmic differential equations in $\mathbb{C}^{2}$ (holomorphic foliations in $\mathbb{P}^{2}$ with the line at infinity invariant).

Proof of Proposition 6.1: Let $\mathcal{M}_{0}(d)$ be the set of all foliations in $\mathcal{M}(d)$ with a center at the origin $(0,0) \in \mathbb{C}^{2} \subset \mathbb{P}^{2}$ and with a local first integral of the type

$$
f=x y+f_{3}+f_{4}+\cdots+f_{n}+\text { h.o.t. }
$$

in a neighborhood of $(0,0)$. Let us prove that $\mathcal{M}_{0}(d)$ is an algebraic subset of $\mathcal{F}(M, L)$.

Let $\mathcal{F}(\omega) \in \mathcal{M}_{0}(d)$ and $\omega=\omega_{1}+\omega_{2}+\omega_{3}+\ldots+\omega_{d+1}$ be the homogeneous decomposition of $\omega$, then in a neighborhood around $(0,0)$ in $\mathbb{C}^{2}$, we have

$$
\omega \wedge d f=0 \Rightarrow\left(\omega_{1}+\omega_{2}+\omega_{3}+\cdots+\omega_{d+1}\right) \wedge\left(d(x y)+d f_{3}+d f_{4}+\cdots\right)=0
$$

Putting the homogeneous parts of the above equation equal to zero, we obtain

$$
\left\{\begin{array}{l}
\omega_{1} \wedge d(x y)=0 \Rightarrow \omega_{1}=k \cdot d(x y), k \text { is constant } \\
\omega_{1} \wedge d f_{3}=-\omega_{2} \wedge d(x y) \\
\cdots \\
\omega_{1} \wedge d f_{n}=-\omega_{2} \wedge d f_{n-1}-\cdots-\omega_{n-1} \wedge d(x y) \\
\cdots
\end{array}\right.
$$

Dividing the 1 -form $\omega$ by $k$, we can assume that $k=1$. Let $\mathcal{P}_{n}$ denote the set of homogeneous polynomials of degree $n$. Define the operator :

$$
\begin{aligned}
S_{n}: \mathcal{P}_{n} & \rightarrow\left(\mathcal{P}_{n} d x \wedge d y\right) \\
S_{n}(g) & =\omega_{1} \wedge d(g)
\end{aligned}
$$


We have

$$
\begin{aligned}
S_{i+j}\left(x^{i} y^{j}\right) & =d(x y) \wedge d\left(x^{i} y^{j}\right)=(x d y+y d x) \wedge\left(x^{i-1} y^{j-1}(j x d y+i y d x)\right) \\
& =(j-i) x^{i} y^{j} d x \wedge d y
\end{aligned}
$$

This implies that when $n$ is odd $S_{n}$ is bijective and so in (6.7), $f_{n}$ is uniquely defined by the terms $f_{m}, \omega_{m}$ 's $m<n$, and when $n$ is even

$$
\operatorname{Im}\left(S_{n}\right)=A_{n} d x \wedge d y
$$

where $A_{n}$ is the subspace generated by the monomials $x^{i} y^{j}, i \neq j$. When $\mathrm{n}$ is even the existence of $f_{n}$ implies that the coefficient of $(x y)^{\frac{n}{2}}$ in

$$
-\omega_{2} \wedge d f_{n-1}-\cdots-\omega_{n-1} \wedge d(x y)
$$

which is a polynomial, say $P_{n}$, with variables

$$
\text { coefficients of } \omega_{2} \ldots \omega_{n-1}, f_{2}, \ldots, f_{n-1}
$$

is zero. The coefficients of $f_{i}, i \leq n-1$ is recursively given as polynomials in coefficients of $\omega_{i}, i \leq n-1$ and so the algebraic set

$$
X: P_{4}=0 \& P_{6}=0 \& \ldots \& P_{n}=0 \ldots
$$

consists of all foliations $\mathcal{F}$ in $\mathcal{F}(M, L)$ which have a formal first integral of the type $(6.6)$ at $(0,0)$. From theorem $\mathrm{A}$ in $[\mathrm{MaMo}]$, it follows that $\mathcal{F}$ has a holomorphic first integral of the type (6.6). This implies that $\mathcal{M}_{0}(d)=X$ is algebraic. Note that by Hilbert zeroes theorem, a finite number of $P_{i}$ 's defines $\mathcal{M}_{0}(d)$. The set $\mathcal{M}(d)$ is obtained by the action of the group of automorphisms of $\mathbb{P}^{2}$ on $\mathcal{M}_{0}(d)$. Since this group is compact we conclude that $\mathcal{M}(d)$ is also algebraic.

\section{Higher Melnikov functions}

In this section we follow the notations introduced in the first lines of Section 4. Let us suppose that $f$ is non composite and $D=M_{\infty}$ is a generic compact $f$-fiber. The integrating factor $s$ of $\omega_{0}$ with

$$
\frac{\omega_{0}}{s}=d f
$$

has $2 D$ as the zero divisor. For simplicity let us write $\omega_{\epsilon}$ instead of $\frac{\omega_{\epsilon}}{s}$ and $\omega_{i}$ instead of $\frac{\omega_{i}}{s}, i=1,2, \ldots$ 
Theorem 7.1 Suppose that $\delta$ is a simple cycle defined in Section 3. If $M_{1} \equiv M_{2} \equiv \cdots \equiv M_{k} \equiv 0$ then

$$
M_{k+1}(t)=-\int_{\delta_{t}}\left(\sum_{i=1}^{k} p_{i} \omega_{k+1-i}+\omega_{k+1}\right)
$$

where $p_{i}$ and $g_{i}$ are meromorphic functions in $M$ with the pole divisors iD and $(i+1) D$, respectively, and are defined recursively by

$$
\omega_{i}+p_{i} d f+d g_{i}=-\sum_{j=1}^{i-1} p_{j} \omega_{i-j}, \quad i=1,2 \ldots, k
$$

Proof: The proof essentially follows [Ro, Proposition 6, p. 73]. We prove by induction on $k$. The case $k=1$ is proved in Proposition 4.1. Let us suppose that the theorem is true for $k-1$, i.e. if $M_{1} \equiv M_{2} \equiv \cdots \equiv M_{k-1} \equiv 0$ then

$$
M_{k}(t)=-\int_{\delta_{t}}\left(\sum_{i=1}^{k-1} p_{i} \omega_{k-i}+\omega_{k}\right)
$$

Now suppose that $M_{k} \equiv 0$. Since $\delta$ is a simple cycle, the 1 -form

$$
-\left(\sum_{i=1}^{k-1} p_{i} \omega_{k-i}+\omega_{k}\right)
$$

is a relatively exact 1 -form with the pole divisor $(k+1) D$ and so by Corollary 5.1 there exist $p_{k}$ and $g_{k}$ with the pole divisors $k D$ and $(k+1) D$, respectively, such that

$$
-\left(\sum_{i=1}^{k-1} p_{i} \omega_{k-i}+\omega_{k}\right)=d g_{k}+p_{k} d f
$$

A direct expansion gives

$$
\left(1+\sum_{i=1}^{k} p_{i} \epsilon^{i}\right) \omega_{\epsilon}=d\left(f-\sum_{i=1}^{k} g_{i} \epsilon^{i}\right)+\left(\sum_{i=1}^{k} p_{i} \omega_{k+1-i}+\omega_{k+1}\right) \epsilon^{k+1}+O\left(\epsilon^{k+2}\right)
$$

Let $\delta_{t, h_{\epsilon}(t)}$ be a path in the leaf of $\mathcal{F}_{\epsilon}$ through $t$ which connects $t$ to $h_{\epsilon}(t)$ along the path $\delta$. Since $\Sigma$ is parameterized by $t=\left.f\right|_{\Sigma}$, integrating the above equality over the path $\delta_{t, h_{\epsilon}(t)}$ we have $\left(h_{\epsilon}(t)-t\right)-\left.\left(\sum_{i=1}^{k} g_{i} \epsilon^{i}\right)\right|_{t} ^{h_{\epsilon}(t)}+\epsilon^{k+1} \int_{\delta_{t, h_{\epsilon}(t)}}\left(\sum_{i=1}^{k} p_{i} \omega_{k+1-i}+\omega_{k+1}\right)+O\left(\epsilon^{k+2}\right)=0$ $\int_{\delta_{t, h_{\epsilon}(t)}}=\int_{\delta_{t}}+O(\epsilon)$ and so by putting zero the coefficient of $\epsilon^{k+1}$ in the above formula we get the desired equality. 


\section{References}

[AB] Anosov, D. V., Bolibruch, A. A.: The Riemann-Hilbert problem. Aspects of Mathematics E22. Friedr. Vieweg \& Sohn, Braunschweig, 1994.

[AGV] Arnold, V. I., Guseïn-Zade, S. M., Varchenko, A. N.: Singularities of differentiable maps. Vol. II. Monodromy and asymptotics of integrals. Monographs in Mathematics 83. Birkhäuser, Boston, MA, 1988.

[Bo] Bonnet, P.: Relative cohomology of polynomial mappings. Manuscripta Math. 110 (2003), no. 4, 413-432.

[Br] Brieskorn, E.: Die Monodromie der isolierten Singularitäten von Hyperflächen. Manuscripta Math. 2 (1970), 103-161.

[CaSa] Camacho, C., SAD, P.: Pontos singulares de equações diferenciais analíticas. $16^{\circ}$ Colóquio Brasileiro de Matemática. Instituto de Matemática Pura e Aplicada, Rio de Janeiro, 1987.

[CeLi] Cerveau, D., Lins Neto, A.: Irreducible components of the space of holomorphic foliations of degree 2 in $\mathbb{P}^{n}, n \geq 3$. Ann. of Math. 143 (1996), $577-612$.

[Ch] ChÉniot, D.: Vanishing cycles in a pencil of hyperplane sections of a nonsingular quasi-projective variety. Proc. London Math. Soc. (3) 72 (1996), no. $3,515-544$.

[Du] Dulac, H.: Sur les cycles limites. Bull. Soc. Math. France 51 (1923), 45-188.

[Ec] ÉCALLE, J.: Introduction aux fonctions analysables et preuve constructive de la conjecture de Dulac. Hermann, Paris, 1992.

[Fr] Francoise, J. P.: Successive derivatives of a first return map, application to the study of quadratic vector fields. Ergodic Theory Dynam. Systems 16 (1996), no. 1, 87-96.

[Ga] Gavrilov, L.: Petrov modules and zeros of Abelian integrals. Bull. Sci. Math. 122 (1998), no. 8, 571-584.

[Go] Gómez-Mont, X.: Integrals for holomorphic foliations with singularities having all leaves compact. Ann. Inst. Fourier (Grenoble) 39 (1989), 451458.

[GrRe] Grauert, H., Remmert, R.: Theorie der Steinschen Räume [Theory of Stein spaces]. Grundlehren der Mathematischen Wissenschaften 227. Springer-Verlag, Berlin-New York, 1977.

[GrRe1] Grauert, H., Remmert, R.: Coherent analytic sheaves. Grundlehren der Mathematischen Wissenschaften 265. Springer-Verlag, Berlin-New York, 1984.

[Il] Ilyashenko, Yu.S.: The appearance of limit cycles under a perturbation of the equation $d w / d z=-R_{z} / R_{w}$, where $R(z, w)$ is a polynomial. Mat. Sb. (N.S.) 78 (120) (1969), 360-373. Translated in: Math. USSR Sbornik 7 (1969), no. 3, 353-364.

[II1] Ilyashenko, YU. S.: Finiteness theorems for limit cycles. Translations of Mathematical Monographs 94. Amer. Math. Soc., Providence, RI, 1991. 
[LP1] Landis, E. M.; Petrovskĭ, I. G.: On the number of limit cycles of the equation $d y / d x=P(x, y) / Q(x, y)$, where $P$ and $Q$ are polynomials. Amer. Math. Soc. Transl. (2) 14 (1960), 181-199.

[LP2] Landis, E. M.; PetrovskiI , I. G.: On the number of limit cycles of the equation $d y / d x=P(x, y) / Q(x, y)$, where $P$ and $Q$ are polynomials of the second degree. 1958 Amer. Math. Soc. Transl. (2) 10 (1958), 177-221.

[Li] Lins Neto, A.; ScÁrdua, B. A.: Foleações Algébricas Complexas, $21^{\circ}$ Colóquio Brasileiro de Matemática.

[Ho1] Movasati, H.: On Deformation of Foliations with a Center in the Projective Space. An. Acad. Brasil. Ciênc. 73 (2001), no. 2, 191-196.

[Ho2] Movasati, H.: On the topology of foliations with a first integral. Bol. Soc. Brasil. Mat. (N.S.) 31 (2000), no. 3, 305-336.

[Ho3] Movasati, H.: Relative cohomology with respect to a Lefschetz Pencil. math. AG/0112204.

[Ho4] Movasati, H.: Center conditions: rigidity of logarithmic differential equations. J. Differential Equations 197 (2004), 197-217.

[La] Lamotke, K.: The topology of complex projective varieties after S. Lefschetz. Topology 20 (1981).

[Ll] Lloyd, N. G.; Pearson, J. M.: Conditions for a centre and the bifurcation of limit cycles in a class of cubic systems. In Bifurcations of planar vector fields (Luminy, 1989), 230-242. Lecture Notes in Math. 1455. Springer, Berlin, 1990.

[Ma] Mardešić, P.: An explicit bound for the multiplicity of zeros of generic Abelian integrals. Nonlinearity 4 (1991), no. 3, 845-852.

[MaMo] Mattei, J.F.; Moussu, R.: Holonomie et integrales premieres. Ann. Sci. École Norm. Sup. (4) 13 (1980), 469-523.

[Mu] MuciÑo, R. J.: Deformations of holomorphic foliations having a meromorphic first integral. J. Reine Angew. Math. 461 (1995), 189-219.

[Po] Poincaré, H.: Sur l'intégration des équations différentielles du premier ordre et du premier degré. Rend. Circ. Mat. Palermo 5 (1891), 161-191.

$[\mathrm{Pu}]$ Pushkar, I. A.: A multidimensional generalization of Ilyashenko's theorem on abelian integrals. Funktsional. Anal. i Prilozhen. 31 (1997), no. 2, 34-44, 95; translation in Funct. Anal. Appl. 31 (1997), no. 2, 100-108.

[Ro] Roussarie, R.: Bifurcation of planar vector fields and Hilbert's sixteenth problem. Progress in Mathematics 164. Birkhäuser Verlag, Basel, 1998.

Recibido: 23 de abril de 2002

Hossein Movasati

Instituto de Matemática Pura e Aplicada, IMPA

Estrada Dona Castorina, 110, 22460-320

Rio de Janeiro, RJ, Brazil

hossein@impa.br 